\title{
LINEARISED EVAPORATION FROM A SOIL OF FINITE DEPTH ABOVE A WATER TABLE
}

\author{
V. T. BUCHWALD ${ }^{1}$ and F. VIERA ${ }^{2}$
}

(Received 28 February 1996; revised 6 March 1997)

\begin{abstract}
The quasi-linear infiltration problem of flow from a semi-infinite wetted region on a soil of finite depth above a horizontal water table is considered in the presence of linearised evaporative loss away from the region. The resulting equations are solved by the WienerHopf technique in terms of certain infinite products. Expressions for the porosity and stream function are derived, and appropriately plotted throughout the layer.
\end{abstract}

\section{Introduction and formulation}

In Cartesian coordinates $(x, y, z)$, with $z$ vertically downwards, the steady flow of water in soils and other porous media is usually described by Darcy's Law (see Philip [6])

$$
\underset{\sim}{F}=-K \nabla(\Psi-z)
$$

where $\underset{\sim}{F}=\theta \underset{\sim}{u}$ is the volumetric flux, $\theta$ is the fraction of the volume occupied by water, $u$ the actual fluid velocity and the factor $K(\theta)$ is the 'hydraulic conductivity'. In this experimental law, $\Psi(\theta)$ is the potential for the local forces (for example, surface tension) arising from the interaction of water, air and soil.

When the soil is fully saturated, then $K=K_{0}$, a constant, but for unsaturated soils $K$ (where $0 \leq K \leq K_{0}$ ) is a function of the moisture content $\theta$, which, in turn is related to the potential $\Psi$. When there is no hysteresis, then $K=K(\Psi)$ may be assumed.

The equation of water conservation is

$$
\underset{\sim}{\nabla} \underset{\sim}{F}=0
$$

\footnotetext{
'Department of Mathematics and Computing Science, The University of the South Pacific, Suva, Fiji, Current address: School of Mathematics, University of New South Wales, Sydney 2052, Australia.

${ }^{2}$ School of Mathematics and Statistics, University of Sydney, NSW 2006.

(C) Australian Mathematical Society, 1998, Serial-fee code 0334-2700/98
} 
so that for steady, saturated flow, $\Psi$ satisfies Laplace's equation, but in unsaturated regions the equation

$$
\underset{\sim}{\nabla} \cdot[K(\Psi) \underset{\sim}{\nabla}(\Psi-z)]=0
$$

is satisfied.

The problem to be considered in this paper follows on from those solved by Weir [12] and Buchwald and Viera [3] so that we adopt their notation by defining the relative permeability $\kappa$ as

$$
\kappa=\mu K / \rho g k_{0},
$$

where $k_{0}$ is the saturation permeability $\left(=\mu K_{0} / \rho g\right), \mu$ is the dynamic viscosity and $\rho$ is the density of water. Consequently $0 \leq \kappa \leq 1, \kappa$ is a measure of wetting and $\kappa=1$ corresponds to a fully wetted soil.

Equation (1.3) is non-linear, but Philip [7] proposes the formula

$$
\kappa=\exp (\alpha \Psi), \quad-\infty<\Psi \leq 0,
$$

as not only a reasonable experimental relation, but also as a transformation of (1.3) which yields the linear equation

$$
\nabla^{2} \kappa-\alpha \frac{\partial \kappa}{\partial z}=0
$$

and the expression

$$
\underset{\sim}{F}=f(\alpha \kappa \underset{\sim}{\hat{z}}-\underset{\sim}{\nabla} \kappa)
$$

for the flux vector, where

$$
f=\rho g k_{0} / \alpha \mu
$$

and $\hat{z}$ is the unit vector vertically downwards. In (1.5), $\alpha$ is a constant with dimension inverse length.

The physical significance of $\alpha$ is fully discussed by Philip [6, 8], who names the quantity $2 \alpha^{-1}$ to be the 'sorptive length'. Briefly, $\alpha$ is proportional to a characteristic capillary pore radius-and for a typical length $L$, the number $\alpha L$ is a measure of the relative importance of gravitational and capillary forces. Philip gives the name 'quasilinear analysis' to the use of (1.5) to derive the linear equation (1.6) by appropriately defining the two material constants $k_{0}$ and $\alpha$.

In the special case of flow in the two dimensions $(x, z),(1.2)$ may be satisfied by assuming a flux stream function $\psi$ such that

$$
\underset{\sim}{F}=f\left(-\frac{\partial \psi}{\partial z}, \frac{\partial \psi}{\partial x}\right)
$$


and then, as was first shown by Raats [10], $\kappa, \psi$ satisfy the generalised CauchyRiemann equations

$$
\begin{aligned}
& \frac{\partial \kappa}{\partial x}-\frac{\partial \psi}{\partial z}=0, \\
& \frac{\partial \kappa}{\partial z}+\frac{\partial \psi}{\partial x}=\alpha \kappa,
\end{aligned}
$$

where both $\kappa$ and $\psi$ satisfy (1.6).

An analogy to wave scattering was pointed out by Waechter and Philip [11] and Philip [9] since (1.6) transforms into a Helmholtz equation. Although this analogy is very effective in some cases, there are two reasons for special care being required in the transition from wave scattering solutions. First, (1.6) is strictly elliptic, so that there are no wave-like solutions, and, moreover, all the uniqueness and extremal properties of solutions to (1.6) are similar to those of Laplace's equation. Second, the restriction $\kappa \leq 1$ is not merely a matter of scaling. For certain boundary value problems the existence of an internal $\kappa=1$ boundary implies a saturated region and two-phase flow. Thus care must be taken to confirm that a formal solution of (1.6) satisfies the condition $0 \leq \kappa \leq 1$.

\section{The infiltration-evaporation problem}

For a soil of infinite depth most of the water descends more or less vertically to infinity, and the question arises as to what happens if the soil is bounded below. Philip [8] has proved that in the absence of sinks there cannot be any steady unsaturated flow when the base is impermeable and horizontal. In our previous paper (Buchwald and Viera, [3] ${ }^{3}$ we showed that above a horizontal impermeable base a steady solution of the unsaturated equations is possible in the presence of a semi-infinite region of evaporation on the surface, the remainder of the surface being wetted by rainfall or irrigation. However, the degree of such wetting was limited by an upper bound, above which the condition $\kappa \leq 1$ was broken in some part of the substrate.

In this paper we consider replacing the impervious base by a water table. The resulting calculations are somewhat similar to BV, but there are physical and mathematical differences which make the investigation worthwhile. In particular, the computed solutions confirm that steady unsaturated flow is possible for all degrees of wetting on the surface.

Let us assume then that there is a water table at $z=d$, and infiltration from constant $\kappa=\kappa_{0} \leq 1$ at $z=0, x<0$, together with evaporation at $z=0, x>0$. We make the 
problem non-dimensional by the transformation

$$
x_{1}=x / d, \quad z_{1}=z / d, \quad \alpha_{1}=\alpha d,
$$

and then, removing the subscript 1 , the boundary value problem, as illustrated in Figure 1, reduces to satisfying (1.6) independent of $y$, with the following boundary conditions at $z=0$ and $z=1$.

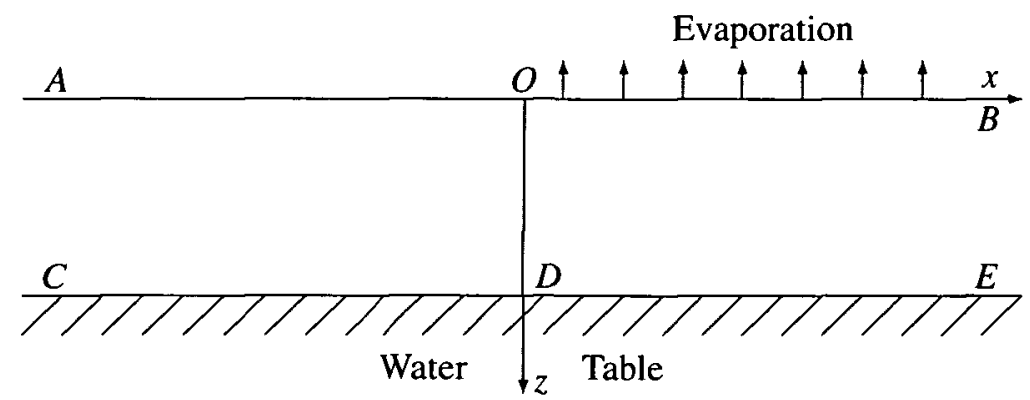

FIGURE 1. A wetted layer at $z=0, x<0$ lies on soil of finite depth above a horizontal water-table at $z=1$. Linear evaporation is assumed at $z=0, x>0$.

From (1.7) the vertical flux $F_{z}$ into the porous medium is

$$
F_{z}=f\left(\alpha \kappa-\frac{\partial \kappa}{\partial z}\right)
$$

where the constant $f$ is defined in (1.8). Following Weir [12], it is assumed that the flux $F_{e}$ due to evaporation out of the material into the atmosphere is described by

$$
F_{e}=h f \kappa, \quad h>0,
$$

across the surface $z=0$. If we assume that evaporation occurs on a portion of this surface, then, equating (2.2) and (2.3), an evaporation condition at $z=0$ is given by

$$
\frac{\partial \kappa}{\partial z}=(h+\alpha) \kappa .
$$

In (2.3) and (2.4) the constant $h$ is a measure of the evaporation, and $h=0$ corresponds to zero flux. We now assume the boundary conditions illustrated in Figure 1:

(i) A wetted region on $A O$, so that

$$
\kappa=\kappa_{0}, \quad z=0, \quad x<0, \quad 0<\kappa_{0} \leq 1,
$$


(ii) evaporation on the surface $O B$, so that (2.4) applies for $z=0, x>0$, and

(iii) there is a water table at $z=1$, so that $\kappa=1$ at $z=1$.

Let

$$
\kappa=\phi(x, z) e^{\beta z},
$$

and then the problem reduces to determining $\phi$ such that

$$
\nabla^{2} \phi-\beta^{2} \phi=0, \quad 0<z<1,
$$

with the boundary conditions

$$
\begin{aligned}
\text { (a) } & \phi=\kappa_{0}, \quad z=0, \quad x<0 ; \\
\text { (b) } & \frac{\partial \phi}{\partial z}-(h+\beta) \phi=0, \quad z=0, \quad x>0 ; \\
\text { (c) } & \phi=\exp (-\beta), \quad z=1, \\
\text { where } & \beta=\frac{1}{2} \alpha>0 .
\end{aligned}
$$

Note that (2.7) is similar to that derived by Waechter and Philip [11].

\section{Behaviour as $|x| \rightarrow \infty$}

(a) $x<0$

It can be shown that the most general solution of (2.7) which satisfies (2.8), (2.10) and is bounded as $x \rightarrow-\infty$ is, for $x<0$, and arbitrary $B_{s}$,

$$
\phi=\phi_{1}+\sum_{s=1}^{\infty} B_{s} e^{\lambda_{s} x} \sin s \pi z,
$$

where

$$
\lambda_{s}=\sqrt{\left(\beta^{2}+s^{2} \pi^{2}\right)},
$$

and

$$
\phi_{1}=A_{1} e^{-\beta z}+A_{2} e^{\beta z},
$$

with

$$
\begin{aligned}
& \left(e^{\alpha}-1\right) A_{1}=\kappa_{0} e^{\alpha}-1, \\
& \left(e^{\alpha}-1\right) A_{2}=1-\kappa_{0} .
\end{aligned}
$$


In the fully saturated case, $\kappa_{0}=1$, and then

$$
A_{1}=1, \quad A_{2}=0 .
$$

Obviously, $\phi \rightarrow \phi_{1}$ as $x \rightarrow-\infty$. It should be noted from (1.7) that the flux due to $\phi_{1}$ is given by

$$
\underset{\sim}{F}=\left(0, f \alpha A_{1}\right),
$$

and this is downwards when $\kappa_{0}>e^{-\alpha}$. When $\kappa_{0}<e^{-\alpha}$ the flux due to $\phi_{1}$ is upwards from the water table to the surface.

(b) $x>0$

The general solution of (2.7) which satisfies (2.9) and (2.10) is, for arbitrary $C_{s}$,

$$
\phi=\phi_{2}+\sum_{s=1}^{\infty} C_{s} e^{-\rho_{s} x} \sin \sigma_{s}(1-z)
$$

where

$$
\begin{aligned}
& \phi_{2}=A_{3} e^{-\beta z}+A_{4} c^{\beta z}, \\
& \rho_{s}=\left(\beta^{2}+\sigma_{s}^{2}\right)^{1 / 2},
\end{aligned}
$$

the $\sigma_{s}$ are the roots of the transcendental equation

$$
\begin{gathered}
c \tan \sigma+\sigma=0, \\
c=h+\beta,
\end{gathered}
$$

and

$$
h^{-1} A_{3}=-(h+\alpha)^{-1} A_{4}=\left[h-(h+\alpha) e^{\alpha}\right]^{-1} .
$$

It may be shown (for example, graphically) that (3.9) has an infinite number of of real roots which have the asymptotic form

$$
\sigma_{s}=\alpha_{s}+c / \alpha_{s}+\mathrm{O}\left(s^{-3}\right)
$$

where

$$
\alpha_{s}=\left(s-\frac{1}{2}\right) \pi
$$

Evidently $\phi \rightarrow \phi_{2}$ as $x \rightarrow \infty$. The flux corresponding to $\phi_{2}$ is $\left(0, f \alpha A_{3}\right)$, and this is always vertically upwards from the water table to the evaporative surface. 


\section{(c) A special case}

Note from (3.7) and (3.11) that at $z=0$,

$$
\kappa_{2}=\phi_{2}(0)=\alpha\left[\alpha e^{\alpha}+h\left(e^{\alpha}-1\right)\right]^{-1}<e^{-\alpha},
$$

and that $\kappa_{2}$ is independent of $\kappa_{0}$, of course.

Thus when $\kappa_{0}=\kappa_{2}$, then necessarily $\phi_{1} \equiv \phi_{2}$ so that in this case

$$
\phi=\phi_{1}
$$

is the complete solution to the problem for all $x$ and the expansions in (3.1) and (3.6) vanish identically.

Thus there are three possible flow regimes for the region $x \rightarrow-\infty$ as follows:

$\mathrm{R} 1: e^{-\alpha}<\kappa_{0} \leq 1$. In this case water flows downwards from the surface to the water table.

$\mathrm{R} 2: \kappa_{2}<\kappa_{0}<e^{-\alpha}$. Water flows upwards, but at a slower rate than in the region $x \rightarrow \infty$.

R3: $\kappa_{0}<\kappa_{2}$. Water flows upwards to the surface faster than in the region $x \rightarrow \infty$.

There are two transition cases. When $\kappa_{0}=e^{-\alpha}$ there is no flow as $x \rightarrow-\infty$. When $\kappa_{0}=\kappa_{2}$, then the flow in the whole region is uniformly upwards and independent of $x$.

\section{The Wiener-Hopf equation}

Let

$$
\Phi=\phi-\phi_{1}
$$

Then, from (3.1), as $x \rightarrow-\infty$,

$$
\Phi=O\left(e^{\lambda_{1} x}\right)
$$

and

$$
\Phi \rightarrow \phi_{2}-\phi_{1}=\mathrm{O}\left(e^{\epsilon x}\right)
$$

as $x \rightarrow \infty$, where $\epsilon>0$ may be taken as small as we like. It is also assumed that $\kappa_{0} \neq \kappa_{2}$, since in that case $\Phi \equiv 0$.

We take the Fourier integral pair

$$
\begin{aligned}
& \hat{\phi}(k, z)=\frac{1}{2 \pi} \int_{-\infty}^{\infty} \Phi(x, z) e^{i k x} d x, \\
& \Phi(x, z)=\int_{-\infty+i \delta}^{\infty+i \delta} \hat{\phi}(k, z) e^{-i k x} d k
\end{aligned}
$$


where the path of integration in (4.4b) is in the strip $S$ for which $\epsilon<\delta<\lambda_{1}$. Substitution into (2.7) yields

$$
\frac{d^{2} \hat{\phi}}{d z^{2}}-\gamma^{2} \hat{\phi}=0
$$

where

$$
\gamma^{2}=\beta^{2}+k^{2}
$$

The general solution of (4.5) is

$$
\hat{\phi}=C(k) \sinh \gamma(1-z)+D(k) \cosh \gamma(1-z) .
$$

By construction, and from (2.10), (3.3), it follows that $\phi=\phi_{1}$ at $z=1$, so that

$$
\Phi(x, 1)=0
$$

Hence, in (4.7), $D \equiv 0$. Noting from (3.3) that $\phi(x, 0)=\phi_{1}(0)$, so that, from (2.8)

$$
\Phi(x, 0)=0, \quad x<0,
$$

and hence, the Fourier transform of $\Phi(x, 0)$ yields

$$
C \sinh \gamma=M^{+}(k),
$$

where, on account of (4.2),

$$
M^{+}(k)=\frac{1}{2 \pi} \int_{0}^{\infty} \Phi(x, 0) e^{i k x} d x
$$

is a function which is analytic for $k$ in the half-plane $S^{+}$for which $\operatorname{Im}(k)>\epsilon$.

Substitution of (4.1) into (2.9) yields the condition that, for $z=0$ and $x>0$,

$$
\frac{\partial \Phi}{\partial z}-c \Phi=c \phi_{1}-\frac{\partial \phi_{1}}{\partial z}=\Phi_{0},
$$

where

$$
\Phi_{0}=(h+\alpha) A_{1}+h A_{2}
$$

and $A_{1}, A_{2}$ are defined in (3.4), (3.5).

The Fourier transform of the left-hand side of (4.12) yields

$$
(\gamma \cosh \gamma+c \sinh \gamma) C=-L^{-}(K)+\frac{\Phi_{0}}{2 \pi i k},
$$


where, since $\Phi(x, 0)=0$ for $x<0$,

$$
L^{-}(k)=\frac{1}{2 \pi} \int_{-\infty}^{0} \frac{\partial \Phi}{\partial z} e^{i k x} d x
$$

is a function which is analytic for $k$ in the half-plane $S^{-}$, for which $\operatorname{Im}(k)<\lambda_{1}$.

Elimination of $C$ from (4.10) and (4.15) yields the Wiener-Hopf equation

$$
K(k) M^{+}(k)+L^{-}(k)=\Phi_{0} / 2 \pi i k,
$$

where

$$
K(k)=(\gamma \cosh \gamma+c \sinh \gamma) / \sinh \gamma,
$$

and (4.16) holds for $k \in S=S^{+} \cap S^{-}$.

In a manner similar to that of BV [3], it is shown in Appendix A that $K(k)$ can be expressed as

$$
K(k)=K(0) P(k) P(-k),
$$

where

$$
K(0)=c+\beta \operatorname{coth} \beta \text {, }
$$

and the infinite product

$$
P(k)=\prod_{s=1}^{\infty}\left\{\left[1-\frac{i k}{\rho_{s}}\right] /\left[1-\frac{i k}{\lambda_{s}}\right]\right\}
$$

with $\rho_{s}, \lambda_{s}$ defined in (3.8), (3.2) respectively, is non-zero, uniformly convergent, and an analytic function of $k$ for $k \in S^{+}$. Similarly $P(-k)$ is non zero and analytic for $k \in S^{-}$.

Now divide (4.16) by $P(-k)$ and let

$$
H^{+}(k)=\frac{\Phi_{0}}{2 \pi i k}, \quad H^{-}(k) \frac{\Phi_{0}}{2 \pi i k}\left\{\frac{1}{P(-k)}-1\right\},
$$

so that $H^{+}(k), H^{-}(k)$ are analytic functions of $k$ for $k \in S^{+}, S^{-}$, respectively. Then (4.16) may be rearranged as

$$
Q(k)=K(0) P(k) M^{+}(k)-H^{+}(k)=H^{-}(k)-L^{-}(k) / P(-k),
$$

for $k \in S$. From (A9) and (A15) of the Appendix it follows that

$$
P(k)=\mathrm{O}\left(|k|^{1 / 2}\right)
$$


as $k \rightarrow \infty$. It is also necessary that $\phi$, and hence $\Phi$ is piece-wise continuous on $z=0$, and hence, from (4.11) and (4.15), $M^{+}(k)=\mathrm{O}\left(k^{-1}\right)$ and $L^{-}(k)=\mathrm{O}(\ln k)$, as $k \rightarrow \infty$. Hence both sides of (4.22) vanish as $k \rightarrow \infty$. Thus $\phi(k) \rightarrow 0$ as $k \rightarrow \infty$ and by analytic continuation, $Q(k)$ is analytic for all $k$. Hence by Liouville's Theorem $Q(k) \equiv 0$, and, therefore, following Noble [5],

$$
\begin{gathered}
K(0) M^{+}(k)=H^{+}(k) / P(k), \\
L^{-}(k)=H^{-}(k) P(-k) .
\end{gathered}
$$

Thus, from (4.4b), (4.7), and (4.24),

$$
\Phi(x, z)=\frac{\Phi_{0}}{2 \pi i K(0)} \int_{-\infty+i \delta}^{\infty+i \delta} \frac{\sinh \gamma(1-z)}{k P(k) \sinh \gamma} e^{-i k x} d k
$$

where $0<\delta<\lambda_{1}$. The expression in (4.26) is suitable for obtaining $\Phi$ by the theorem of residues for $x<0$. An alternative expression, obtained by using (4.17) and (4.18), is

$$
\Phi(x, z)=\frac{\Phi_{0}}{2 \pi i} \int_{-\infty+i \delta}^{\infty+i \delta} \frac{P(-k) \sinh \gamma(1-z)}{k(\gamma \cosh \gamma+c \sinh \gamma)} e^{-i k x} d k
$$

and this is suitable for evaluation for $x>0$.

Noting that

$$
\kappa=\left(\Phi+\phi_{1}\right) e^{\beta z}
$$

expressions for the stream function $\psi$ are obtained from (1.10) and (1.11), so that apart from an arbitrary constant,

$$
\psi(x, z)=\alpha A_{1} x+\frac{\Phi_{0} e^{\beta z}}{2 \pi K(0)} \int_{-\infty+i \delta}^{\infty+i \delta} \frac{\Gamma(x, z)}{k P(k) \sinh \gamma} d k,
$$

where

$$
\Gamma(x, z)=[\beta \sinh \gamma(1-z)+\gamma \cosh \gamma(1-z)] e^{-i k x} .
$$

Here again, the result in (4.29) is suitable for computations for $x<0$, and the equivalent formula for $x>0$ is

$$
\psi(x, z)=\alpha A_{1} x+\frac{\Phi_{0} e^{\beta z}}{2 \pi} \int_{-\infty+i \delta}^{\infty+i \delta} \frac{\Gamma(x, z) P(-k)}{k^{2}(\gamma \cosh \gamma+c \sinh \gamma)} d k
$$




\section{The singularity at $(0,0)$}

As a first step consider the result in (4.26) in the limit as $z \rightarrow 0$ and $\delta \rightarrow 0$. Then

$$
\Phi(x, 0)=\frac{\Phi_{0} Y(x)}{2 \pi i K(0)}
$$

where

$$
Y(x)=\int_{-\infty}^{\infty} \frac{e^{-i k x}}{k P(k)} d k
$$

is part of a Fourier transform pair defined in the generalised sense, (Lighthill [4]), and $Y(x)$ has a singularity only at $x=0$. The nature of this singularity is determined by an inverse method.

Using the notation of (A4) and (A10).

$$
K(k)=f_{2}(k) / f_{1}(k)
$$

and hence

$$
P(k)=G(k) / F(k)
$$

with $K(0)=f_{2}(0) / f_{1}(0)$. Hence, from (A9) and $\mathrm{A}(15)$

$$
P(k) \sim\{k / K(0)\}^{1 / 2} e^{i \pi / 4},
$$

as $k \rightarrow \infty$. By definition $P(k)$ is analytic for $\operatorname{Im}(k) \geq 0$, so that, necessarily

$$
P(k) \sim\{|k| / K(0)\}^{1 / 2} e^{i \pi},
$$

as $k \rightarrow-\infty$. Combining (5.5) and (5.6), it follows that in (5.2)

$$
\frac{1}{k P(k)} \sim-i[K(0)]^{1 / 2}|k|^{-3 / 2} e^{3 \pi i / 4 \operatorname{sgn} k}
$$

as $k \rightarrow \pm \infty$. Since $Y(x)$ has a singularity at $x=0$ only, comparison with Table 1 of [4] shows that, as $x \rightarrow 0$,

$$
Y(x) \sim-4 i\{\pi x K(0)\}^{1 / 2} H(x),
$$

where $H(x)$ is the Heaviside unit function, so that, in (5.1)

$$
\begin{aligned}
\Phi(x, 0) & \sim-2 \Phi_{0}\left\{\frac{x}{\pi K(0)}\right\}^{1 / 2} H(x), \\
& =-S x^{1 / 2} H(x), \text { say. }
\end{aligned}
$$


For the next step it should be noted that $\Phi$ satisfies (2.7). Let $(r, \phi)$ be polar coordinates centred at the origin, so that $r=\left(x^{2}+z^{2}\right)^{1 / 2}$. Dimensional analysis indicates that, $\beta r \rightarrow 0$,

$$
\Phi \sim \Phi_{s},
$$

where $\Phi_{s}$ satisfies Laplace's equation

$$
\nabla^{2} \Phi_{s}=0
$$

Hence $\Phi_{s}$ and the corresponding stream function $\Psi$ are the real and imaginary parts of a function of the complex variable $\xi=x+i z$. Thus

$$
\Phi_{s}+i \Psi_{s}=-S \xi^{1 / 2}
$$

Inspection shows that for $z=0$, the result in (5.12) is consistent with (5.10). Let $\xi=r e^{i \theta}$, and then, in polar coordinates, as $r \rightarrow 0$,

$$
\begin{aligned}
& \phi-\phi_{1} \sim-S r^{1 / 2} \cos \frac{1}{2} \theta, \\
& \psi-\psi_{1} \sim-S r^{1 / 2} \sin \frac{1}{2} \theta .
\end{aligned}
$$

In (5.13) and (5.14), $\phi_{1}$ is given in (3.3), and $\psi_{1}$ is from (1.10), (1.11) to be given by

$$
\psi_{1}=2 \beta A_{1} x
$$

\section{Results}

For $0 \leq z \leq 1$ the integrals in (4.26) - (4.31) are $\mathrm{O}\left(k^{-3 / 2}\right)$ as $k \rightarrow \infty$ so that the integrals may be evaluated by using Jordan's Lemma, and summing residues at the poles in $S^{+}$for $x<0$, and in $S^{-}$for $x>0$.

For $x<0$ we use (4.26) and (4.29), for which the poles in $S^{+}$are at

$$
\gamma=n \pi i, \quad k=\lambda_{n} i=\left(\beta^{2}+n^{2} \pi^{2}\right)^{1 / 2} i,
$$

with the results that, for $x<0$,

$$
\kappa=A_{1}+A_{2} e^{2 \beta z}-\frac{\pi \Phi_{0} e^{\beta z}}{K(0)} \sum_{n=1}^{\infty} \frac{n \sin n \pi z}{\lambda_{n}^{2} P\left(i \lambda_{n}\right)} e^{\lambda_{n} x},
$$




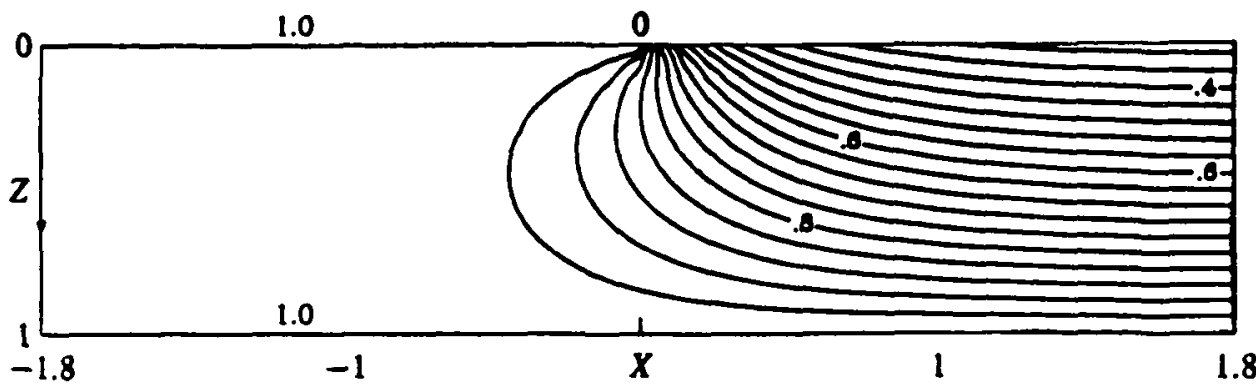

(a)

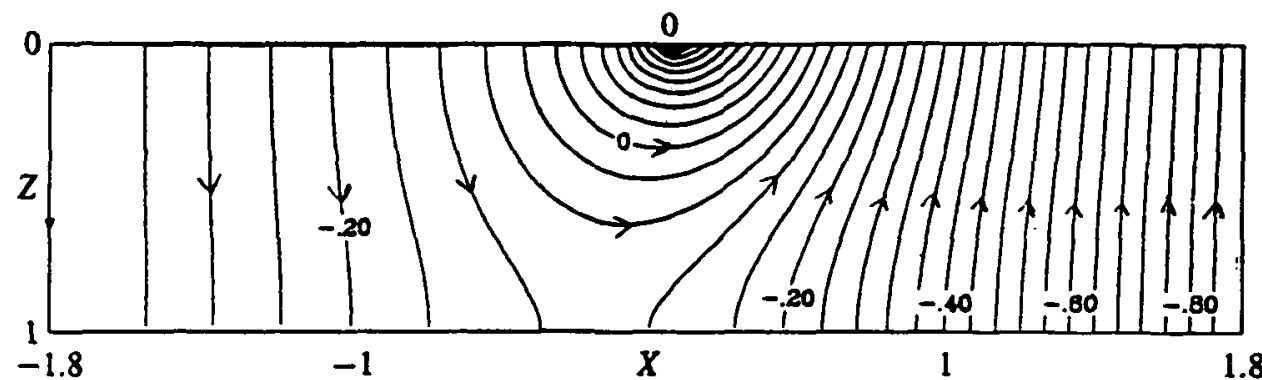

(b)

FIGURE 2. Contour lines for (a) the relative permeability $\kappa$ and (b) the stream function $\psi$ for $h=2$, $\beta=0.1$ and $\kappa_{0}=1$. Note that $\kappa \approx 1$ for $x<0$, and that the flow is downward for $x<0$ and upward for $x>0$.

and

$$
\psi=\alpha A_{1} x+\frac{\pi \Phi_{0} e^{\beta z}}{K(0)} \sum_{n=1}^{\infty} \frac{n(n \pi \cos n \pi z-\beta \sin n \pi z) e^{\lambda_{n} x}}{\lambda_{n}^{3} P\left(i \lambda_{n}\right)}
$$

In (6.2) and (6.3), $A_{1}, A_{2}$ are given in (3.4), (3.5), $\Phi_{0}$ in (4.13), $K(0)$ in (4.19) and the infinite product $P(k)$ in (4.20).

For $x>0$, the singularities in $S^{-}$are poles at $k=0$, and $k=-i \rho_{n}, \gamma=i \sigma_{n}$, where $\rho_{n}, \sigma_{n}$ are determined from (3.8) and (3.9). In (4.27), all the poles are simple, and the result is that, for $x>0$,

$$
\kappa=\phi_{2} e^{\beta z}+\Phi_{0} e^{\beta z} \sum_{n=1}^{\infty} \frac{\sigma_{n}^{2} P\left(i \rho_{n}\right) \sin \sigma_{n}(1-z)}{\rho_{n}^{2}\left\{c(1+c)+\sigma_{n}^{2}\right\} \sin \sigma_{n}} e^{-\rho_{n} x},
$$

where $\phi_{2}$ is defined in (3.7) and (3.11).

In the expression for $\psi$ in (4.31) there is a double pole at $k=0$, the contribution from which is

$$
\psi_{0}=\left(i P^{\prime}(0)-x\right) H \Phi_{0}
$$




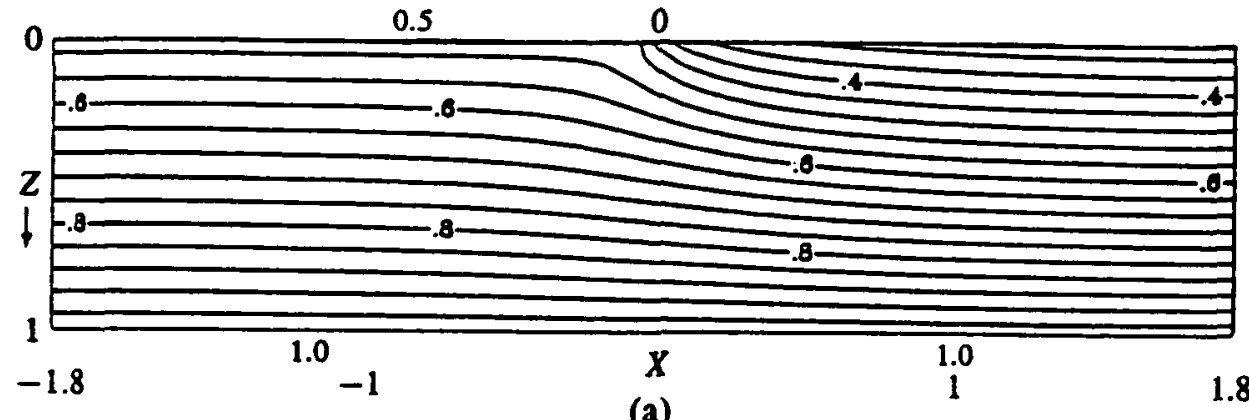

(a)

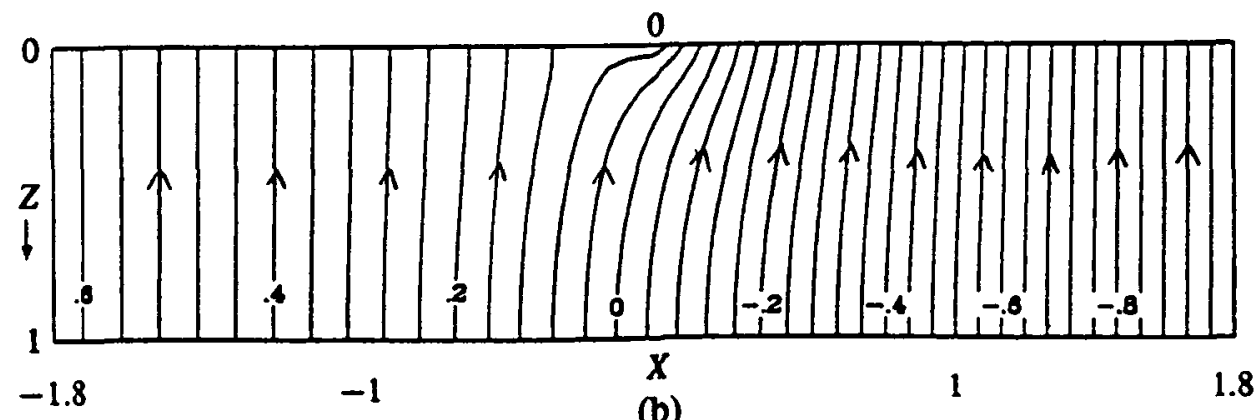

(b)

FIGURE 3. As for Figure 2, but with $\kappa_{0}=0.5$. This case is in the region $R_{2}$, so that flow is everywhere upward, but faster for $x>0$. Note that the stream function contours are closer for $x>0$.

where

$$
H=\alpha /\left(h+\alpha-h e^{-\alpha}\right) .
$$

The result is that, for $x>0$,

$$
\psi=M+\alpha A_{3} x-\Phi_{0} e^{\beta z} \sum_{n=1}^{\infty} \frac{\sigma_{n}^{2} P\left(i \rho_{n}\right)\left[\sigma_{n} \cos \sigma_{n}(1-z)+\beta \sin \sigma_{n}(1-z)\right]}{\rho_{n}^{3}\left(c+c^{2}+\sigma_{n}^{2}\right) \sin \sigma_{n}} e^{-\rho_{n} x}
$$

and

$$
M=\Phi_{0} H \sum_{n=1}^{\infty}\left(\frac{1}{\rho_{n}}-\frac{1}{\lambda_{n}}\right)
$$

The sum in (6.8), obtained by differentiating the logarithm of (4.20), converges only slowly. A quick method of finding this sum is given in Appendix B.

Using (6.2), (6.3), (6.4) and (6.7) numerical values of the relative porosity $\kappa$ and the stream function $\psi$ were obtained as functions of $(x, z)$ for various values of the parameters $h, \beta$ and $\kappa_{0}$. It is conjectured from the physics of the problem that even for 


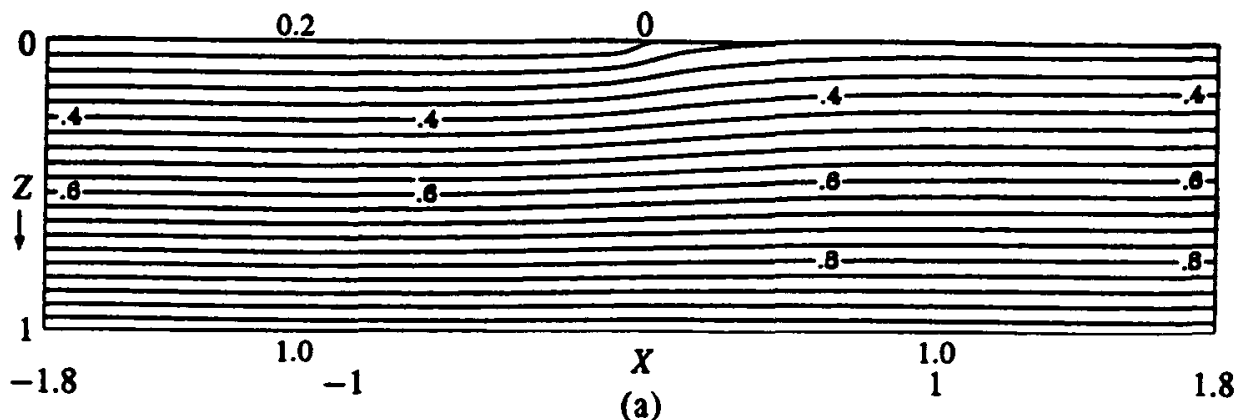

(a)

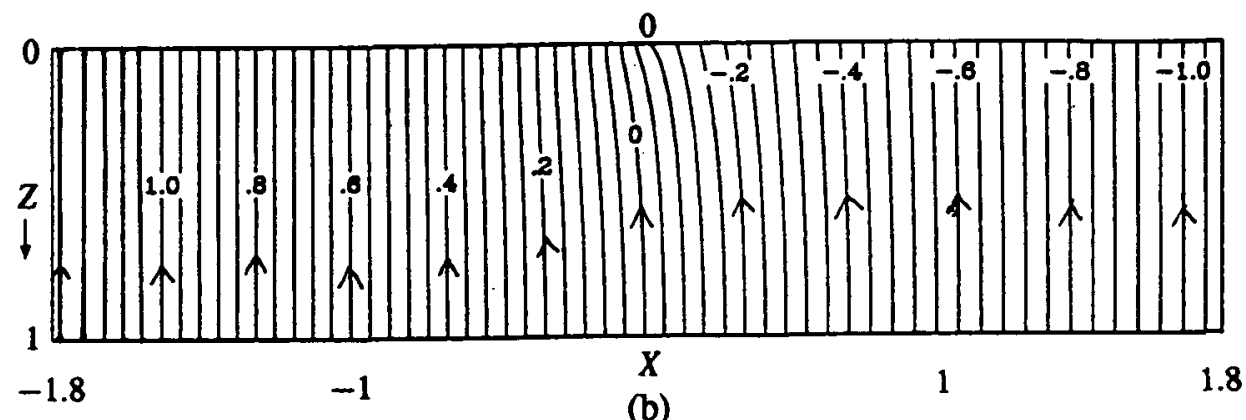

FIGURE 4. As for Figure 2 and 3, but with $\kappa_{0}=0.2$, so that upward flow is faster for $x<0$.

$\kappa_{0}=1$ (that is, a fully saturated boundary at $z=0, x=0$ ) the quasi-linear equations have a solution which satisfies $\kappa \leq 1$ everywhere in the layer, and this corresponds to a physically acceptable solution for all values of the parameters. The numerical results so far obtained for a range parameter values are consistent with this conjecture.

A selection of numerical results is presented in the figures which illustrates the three flow regimes $R_{1}: e^{-\alpha}<\kappa_{0} \leq 1, R_{2}: \kappa_{2}<\kappa_{0}<e^{-\alpha}$ and $R_{3}: \kappa_{0} \leq \kappa_{2}$. The first calculations take $(h, \beta)=(2,0.1)$, and then choosing $\kappa_{0}=1,0.5$ and 0.2 yields flow in the regions $R_{1}, R_{2}$ and $R_{3}$, respectively. These cases are illustrated in Figures $2-4$. As expected, there is strong downward flow for $x<0$ in Figure 2, and upward flow for $x>0$, with a transition region near $x=0$.

The effect of a decrease in the sorptive length $\beta^{-1}$ is illustrated in Figure 5, in which $\beta=0.4, h=2$ and $\kappa_{0}=1$. Comparison with Figure 2 shows a-stronger downward flow in $x<0$, and consequent extra penetration of the flow from the wetted region into $x>0$.

In Figure 6 the effect of decreasing the evaporation is shown. Comparison of Figure $6 \mathrm{~b}$ with Figure $5 \mathrm{~b}$ shows deep penetration from the wetted region into $x>0$. This penetration will increase as $h$ decreases. 

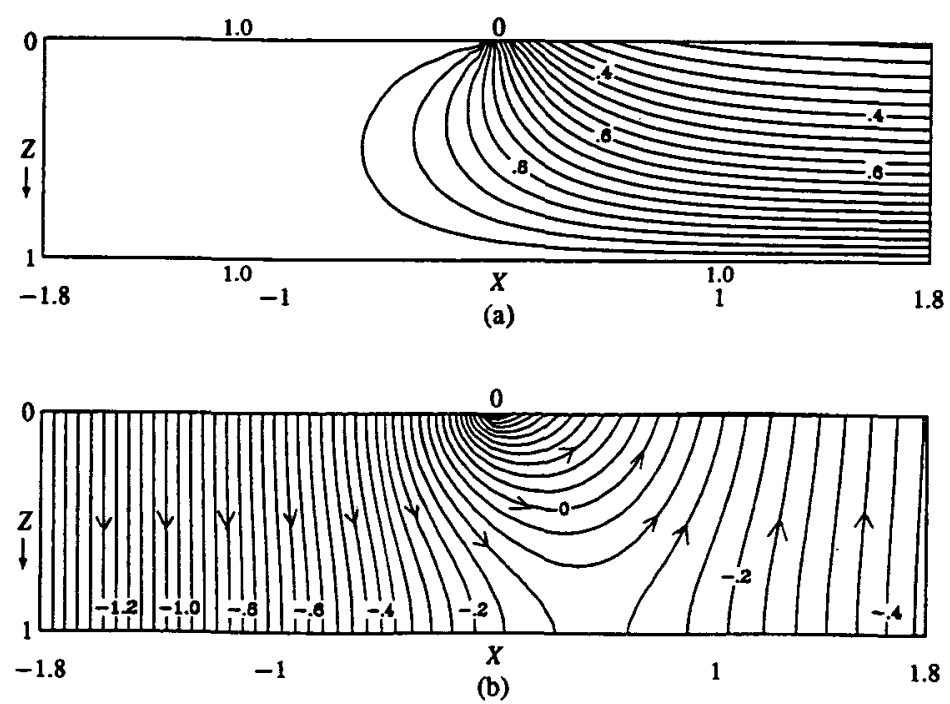

FIGURE 5. This figure should be compared with Figure 2, with $h=2, \kappa_{0}=1$, but $\beta$ having the increased value of 0.4 .
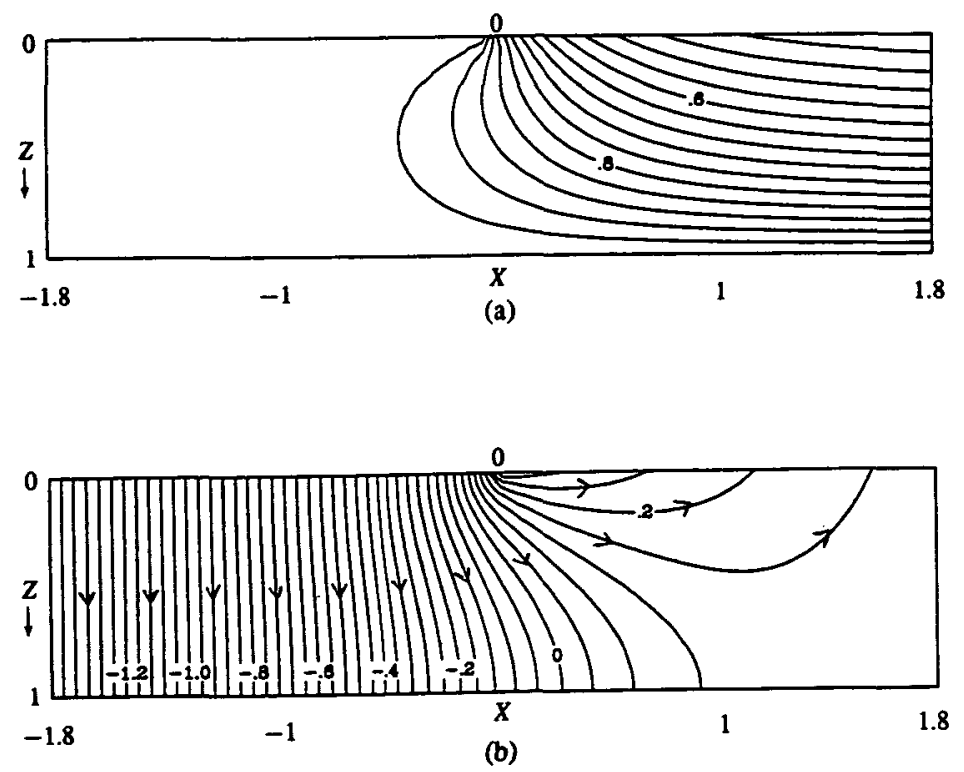

FIGURE 6. Relative permeability and stream function for $\kappa_{0}=1, \beta=0.4$ as in Figure 5, but with reduced evaporation $h=0.2$. 


\section{Acknowledgement}

This work was supported by an Institutional Grant from the Australian Research Council through the University of Sydney.

\section{Appendix A}

For $\beta>0$, and

$$
\gamma=\left(\beta^{2}+k^{2}\right)^{1 / 2}
$$

the even entire function

$$
f_{1}(k)=\gamma^{-1} \sinh \gamma
$$

has zeros at $k= \pm i \lambda_{n}$, where

$$
\lambda_{n}=\left(\beta^{2}+n^{2} \pi^{2}\right)^{1 / 2}, \quad n=1,2,3, \ldots,
$$

Hence, by a well known factorisation theorem (Whittaker and Watson, [13])

$$
f_{1}(k)=f_{1}(0) F(k) F(-k),
$$

where

$$
F(k)=e^{-v_{0} i k / \pi} \prod_{n=1}^{\infty}\left(1-\frac{i k}{\lambda_{n}}\right) e^{i k / n \pi}
$$

is analytic and non-zero for $\operatorname{Im}(k)>-\lambda_{1}$, since $\lambda_{n} \sim n \pi$ as $n \rightarrow \infty$ so that the infinite product in (A5) is uniformly convergent. Also $F(-k)$ is analytic and non-zero for $\operatorname{Im}(k)<\lambda_{1}$. In (A5), $v_{0}$ is Euler's constant.

From the Weierstrass definition of the factorial function (Abramowitz and Stegun, [1]).

$$
F_{0}(k)=\{(-i k / \pi) !\}^{-1}=e^{-v_{0} i k / \pi} \prod_{n=1}^{\infty}\left(1-\frac{i k}{n \pi}\right) e^{i k / n \pi} .
$$

After some rearrangement it follows that

$$
F(k) / F_{0}(k)=\prod_{n=1}^{\infty}\left\{\frac{n \pi}{\lambda_{n}}\right\} \prod_{n=1}^{\infty}\left\{1-\frac{\lambda_{n}-n \pi}{n \pi-i k}\right\} .
$$


Since $\lambda_{n}=n \pi+\mathrm{O}\left(n^{-1}\right)$ as $n \rightarrow \infty$, the second infinite product in (A7) is uniformly convergent, and tends to 1 as $k \rightarrow \infty$. Moreover

$$
\prod_{n=1}^{\infty} \frac{n^{2} \pi^{2}}{\lambda_{n}^{2}}=\beta / \sinh \beta,
$$

so that

$$
F(k) / F_{0}(k) \sim\left\{f_{1}(0)\right\}^{-1 / 2},
$$

as $k \rightarrow \infty$. By Stirling's formula, then,

$$
F(k) \sim\left\{2 k f_{1}(0)\right\}^{-1 / 2}\left(-\frac{i k}{e \pi}\right)^{i k / \pi} e^{i \pi / 4},
$$

as $k \rightarrow \infty$. Similarly, let

$$
f_{2}(k)=\cosh \gamma+c \gamma^{-1} \sinh \gamma,
$$

and then, following BV [3] (equations 5.1-5.7) and Buchwald [2],

$$
f_{2}(k)=f_{2}(0) G(k) G(-k),
$$

where

$$
G(k)=e^{-\nu_{0} i k / \pi} \prod_{n=1}^{\infty}\left[1-\frac{i k}{\rho_{n}}\right] e^{i k / n \pi},
$$

and

$$
\rho_{n}=\left(\beta^{2}+\sigma_{n}^{2}\right)^{1 / 2}
$$

where the $\sigma_{n}$ are the non trivial roots of

$$
c \tan \sigma+\sigma=0
$$

Note that $G(k), G(-k)$ are analytic and non-zero for $\operatorname{Im}(k)>-\rho_{1}, \operatorname{Im}(k)<\rho_{1}$, respectively. Moreover, as $k \rightarrow \infty$,

$$
G(k) \sim\left\{2 f_{2}(0)\right\}^{-1 / 2}\left\{-\frac{i k}{\pi e}\right\}^{i k / \pi} .
$$




\section{Appendix B}

Since the series in (5.8) is absolutely convergent, we rewrite (5.8) in the form

$$
\begin{aligned}
\Phi_{0}^{-1} H^{-1} C= & \sum_{n=1}^{\infty}\left[\frac{1}{\rho_{n}}-\frac{2}{\pi(2 n-1)}\right] \\
& -\sum_{n=1}^{\infty}\left[\frac{1}{\lambda_{n}}-\frac{1}{n \pi}\right]+\frac{1}{\pi} \sum_{n=1}^{\infty}\left[\frac{2}{2 n-1}-\frac{1}{n}\right] .
\end{aligned}
$$

The series

$$
\sum_{n=1}^{\infty}\left[\frac{2}{2 n-1}-\frac{1}{n}\right]=2 \ln 2,
$$

and from (1.15) and (1.25),

$$
\lambda_{n}=n \pi+\mathrm{O}\left(n^{-1}\right), \quad \rho_{n}=\left(n-\frac{1}{2}\right) \pi+\mathrm{O}\left(n^{-1}\right),
$$

as $n \rightarrow \infty$, so that the terms in each of the first two series in (B1) are $\mathrm{O}\left(n^{-3}\right)$ as $n \rightarrow \infty$, which is rapid enough convergence to make computation feasible.

\section{References}

[1] M. Abramowitz and I. A. Stegun (eds). Handbook of mathematical functions, (Dover, New York, 1970).

[2] V. T. Buchwald, "The value of certain infinite products" Gazette Aust. Math. Soc. 21 (1994) 39-40.

[3] V. T. Buchwald and F. Viera, "Linearised evaporation from a soil of finite depth near a wetted region," Quart. J. Mech. App. Math. 49 (1996) 49-64.

[4] M. J. Lighthill, "Introduction to Fourier analysis and generalised functions," (Cambridge Univ. Press, Cambridge, 1960).

[5] B. Noble, Methods based on the Wiener-Hopf technique (Pergamon Press, London, 1958, Example 3.4).

[6] J. R. Philip, "Flow in porous media", Annual Rev. Fluid Mech. 2 (1970) 177-204.

[7] J. R. Philip, "Aspects of quasilinear infiltration from surface sources, especially the case $\alpha=0$." Water Resources Res. 20 (1984) 633-635.

[8] J. R. Philip, "Steady unsaturated seepage above a sloping impermeable base", Water Resources Res. 24 (1988) 1192-1196.

[9] J. R. Philip "The scattering analog for infiltration in porous media." Rev. Geophys. 27 (1989) $431-448$.

[10] P. A. C. Raats, "Steady infiltration from line sources and furrows" Soil Sci. Soc. Am. Proc. 34 (1970) 709-714.

[11] R. T. Waechter and J. R. Philip, "Steady two and three dimensional flows in unsaturated soil: the scattering analog" Water Resources Res. 21 (1985) 1875-1887. 
[12] G. J. Weir, "Linearised evaporation about a shallow half-plane pond", J. Aust. Math. Soc. Ser. B, 34 (1993) 355-367.

[13] E. T. Whittaker and G. N. Watson, A course of modern analysis, (Cambridge Univ. Press, Cambridge, 1963). 